\title{
Crescimento de genótipos diplóides de bananeira submetidos ao estresse salino
}

\author{
Gilberto de S. E S. Junior ${ }^{1}$, Marciana B. de Morais ${ }^{2}$, \\ Terezinha R. Camara ${ }^{3} \&$ Lilia Willadino ${ }^{4}$
}

\begin{abstract}
RESU M 0
Neste trabalho foram avaliados dez genótipos diplóides de bananeira (Musa spp) quanto a tolerância à salinidade, estresse abiótico que limita a produtividade da cultura. As plantas foram cultivadas durante 21 dias, em solução acrescida ou não de $100 \mathrm{~mol} \mathrm{~m}^{-3} \mathrm{de} \mathrm{NaCl}$ e analisadas variáveis de crescimento que incluem área foliar, biomassa fresca e seca, alocação de biomassa e taxa de crescimento. 0 experimento foi conduzido em delineamento inteiramente casualizado em fatorial $10 \times 2$ e três repeticões. $\mathrm{N}$ a maioria dos genótipos estudados a salinidade provocou reduções em quase todas as variáveis analisadas. 0 genótipo Lidi destacou-se por apresentar melhor adaptação ao estresse salino, em todas as variáveis biométricas e capacidade de manutenção, sob estresse, da biomassa seca e fresca (limbo, caule, pseudocaule + raiz), área foliar, além de taxa de crescimento absoluto, entre outros. A produção relativa da biomassa seca da parte aérea foi superior a $70 \%$, caracterizando este genótipo como tolerante e promissor para ser integrado a programas de melhoramento. O s genótipos 0 uro e Tungia, por sua vez, sofreram grande redução da taxa de crescimento absoluto e a produção relativa da biomassa seca foi inferior a $50 \%$ caracterizando este genótipo como sensivel à salinidade.
\end{abstract}

Palavras-chave: Musa spp., área foliar, biomassa seca, taxa de crescimento

\section{Growth of dipoid banana genotypes under saline stress}

\begin{abstract}
Ten diploid banana genotypes (Musa spp.) were evaluated with regard to salt tolerance, abiotic stress which limits the productivity of the crop. The plants were grow $n$ during 21 days, in a nutrient solution with and without the addition of $\mathrm{NaCl}\left(0\right.$ and $\left.100 \mathrm{~mol} \mathrm{~m}^{-3}\right)$. Growth variables including leaf area, fresh and dry biomass, biomass allocation and growth rate were analysed. The experiment was implemented in a completely randomized design in factorial $(10 \times 2)$ arrangement and three replicates per treatment. Salinity caused in most genotypes reductions in almost all variables. Genotype Lidi stood out by having better adaptation to saline stress in all measured biometric variables with the ability to maintain, under stress, the fresh and dry biomass (leaf blade, stem, pseudostem + root), leaf area, and absolute growth rate among others. The relative production of dry biomass of shoots was more than $70 \%$, characterizing this as a tolerant genotype and promising to be integrated into breeding programs. Genotypes 0 uro and Tungia, in turn, showed reduction of absolute grow th rate and relative dry biomass production (less than $50 \%$ ) were characterized as sensitive to salinity.
\end{abstract}

Key words: Musa spp., leaf area, dry biomass, growth rate

\footnotetext{
${ }^{1}$ Instituto Federal de Educação, Ciência e Tecnologia de Pernambuco, Avenida Professor Luiz Freire, 500, Cidade U niversitária, CEP 52740-540, Recife, PE. E-mail:gilbertojunior26@yahoo.com.br

${ }^{2}$ M estranda Depto Agronomia, U FRPE, Rua D. Manoel de M edeiros s/n, D ois Irmãos. CEP: 52171-900 Recife, PE. E-mail: marciana.bio@gmail.com 3 DQ/UFRPE. E-mail: tkrcamara@bol.com.br

${ }^{4}$ D B/U FRPE e Instituto N acional de Ciência e Tecnologia em Salinidade (IN CTSal/CN Pq). Fone: (81) 3320 6366. E-mail: willadino.lilia@gmail.com
} 


\section{INTRODUÇÃO}

A bananeira (Musa spp) está entre as culturas agrícolas mais importantes nas regiões tropicais e subtropicais do mundo abrangendo mais de 115 países. Abananicultura é uma atividade que apresenta uma produção mundial de cerca de 95 milhões de toneladas de fruta fresca, em área colhida de 4,8 milhões de hectares (Silva Neto \& Guimarães, 2011). Dentre as frutíferas a banana ocupa a segunda posição na produção mundial, superada apenas pela melancia (FAO/STAT, 2011). No Brasil, a área cultivada com bananeiras passou, ao longo dos últimos 30 anos, de 343,6 mil hectares para 511,6 mil hectares, representando $49 \%$ de aumento (Silva Neto \& Guimarães, 2011).

A cultura da bananeira apresenta significativa limitação de produção quando cultivada em áreas salinizadas. Associada à sodicidade, a salinidade do solo é um problema de extensão mundial. Atualmente, cerca de 900 milhões de hectares estão afetados pelos sais (Fageria et al., 2010). A salinização dos solos ocorre, sobremaneira, nas regiões de clima árido e semiárido, onde se constitui em fator limitante da produção agrícola. Nesses ambientes as principais causas dos processos de salinização das áreas agricultáveis são decorrentes da baixa precipitação pluviométrica, alta evaporação, material de origem dos solos e irrigação, além de drenagem inadequada (Dasgan et al., 2002).

Em diversos países as áreas salinizadas vêm sendo exploradas com sucesso graças à utilização de espécies tolerantes à salinidade. $\mathrm{O}$ aumento da produtividade e da qualidade da banana, através da introdução de materiais tolerantes, tende a favorecer o aumento nas exportações do produto e contribui para superação das disparidades regionais. Assim, a caracterização e avaliação de genótipos diploides de bananeira, que são fontes de genes de interesse para os programas de melhoramento vegetal, são etapas imprescindíveis na utilização do banco de germoplasma permitindo identificar genótipos promissores para que possam ser integrados aos programas de melhoramento genético (Gomes et al., 2004).

Segundo Benincasa (2003) a análise de crescimento é o método mais acessível e preciso para avaliar o crescimento de plantas e inferir a contribuição dos diversos processos fisiológicos sobre o comportamento das mesmas. O estado fenológico e os níveis de salinidade aplicados durante o crescimento e desenvolvimento dos genótipos sob avaliação são de fundamental importância no processo de geração de cultivares tolerantes (Fageria et al., 2010). A produção relativa de biomassa fresca ou seca tem sido indicada como um dos parâmetros mais realísticos de tolerância, haja vista sua relação significativa com a produção (Aslam et al., 1993).

Selecionar genótipos diploides de bananeira capazes de produzir respostas indicadoras de tolerância em ambientes salinos é uma tarefa complexa, nas quais interagem variáveis fisiológicas, bioquímicas e moleculares. Mesmo assim é possível selecionar, dentre um banco de germoplasma, aqueles materiais mais tolerantes à salinidade e que, ao mesmo tempo, apresentem uma produção maior de biomassa seca (Gomes et al., 2004; 2005b).
Com base no exposto objetivou-se selecionar genótipos diploides de bananeira com tolerância diferenciada à salinidade utilizando-se diferentes variáveis de crescimento.

\section{Material e MÉTODOS}

O experimento foi conduzido em casa de vegetação na Universidade Rural Federal de Pernambuco, latitude $8^{\circ} 04^{\prime}$ 03' $\mathrm{S}$ e longitude $34^{\circ} 55^{\prime} 00^{\prime}$ ' W. Dez genótipos diploides de bananeira foram avaliados, quais sejam: Nyarmo Yik, Thong Dok Mak, Berlim, Pisang Ceylan, Tungia, Madu, Lidi, Ouro, Malbut e Calcuttá (Tabela 1). Mudas provenientes da micropropagação, com aproximadamente $15,0 \mathrm{~cm}$ de altura obtidas do banco de germoplasma do Centro Nacional de Pesquisa Mandioca e Fruticultura Tropical -CNPMF/EMBRAPA, BA, foram plantadas em sacos de polietileno contendo $10 \mathrm{~kg}$ de areia lavada.

Tabela 1. Relação dos genótipos obtidos do banco de germoplasma do CN PM F/EM BRAPA - BA, utilizados no experimento

\begin{tabular}{|c|c|c|c|}
\hline $\begin{array}{l}\text { Nome do } \\
\text { acesso }\end{array}$ & Código & Sinonímia & $\begin{array}{c}\text { Origem/ } \\
\text { Procedência }\end{array}$ \\
\hline NyarmoYik & BRA002984/SF248 & - & Nova Guiné \\
\hline Thong DokMak & BRA004472/SF712 & Palen Berry & Tailândia \\
\hline Berlim & BRA004952/SF907 & Trimulin & Indonésia \\
\hline PisangCeylan & - & - & - \\
\hline Tungia & - & - & Indonésia \\
\hline Madu & - & - & Honduras \\
\hline Lidi & - & Lilim & Honduras \\
\hline Ouro & BRA003042/SF286 & Kirun & Nova Guiné \\
\hline Malbut & BRA002674/SF217 & - & Nova Guiné \\
\hline Calcuttá & - & Burmannica & Jamaica \\
\hline
\end{tabular}

Fonte: Silva et al. (1999)

Durante o período de restabelecimento do estresse do plantio as plantas foram irrigadas diariamente com solução nutritiva contendo $742,86 \mathrm{mg} \mathrm{L}^{-1}$ de fertilizante solúvel com a seguinte composição: $3 \% \mathrm{~N}, 11 \% \mathrm{P}_{2} \mathrm{O}_{5}, 38 \% \mathrm{~K}_{2} \mathrm{O}, 4 \% \mathrm{MgO}$, $11 \%$ S, $0,025 \%$ B, 0,004\% Mo, 0,01\% Cu-EDTA, 0, $025 \%$ ZnEDTA, 0,07\% Fe-EDTA e 0,04\% Mn-EDTA e 840,00 $\mathrm{mg} \mathrm{L}^{-1} \mathrm{de}$ nitrato de cálcio $(15,5 \% \mathrm{Ne} 19,0 \% \mathrm{Ca})$.

Após o período de aclimatação as mudas foram submetidas a dois tratamentos: 0 e $100 \mathrm{~mol} \mathrm{~m}^{-3}$ de $\mathrm{NaCl}$. O estabelecimento do tratamento com $100 \mathrm{~mol} \mathrm{~m}^{-3} \mathrm{de} \mathrm{NaCl}$ foi realizado em duas etapas para evitar o choque osmótico nas mudas: inicialmente, foi aplicada uma solução de $50 \mathrm{~mol} \mathrm{~m}^{-3} \mathrm{de} \mathrm{NaCl}$ e após uma semana a concentração foi aumentada para $100 \mathrm{~mol} \mathrm{~m}^{-3}$. A média da condutividade elétrica das soluções nutritivas $\left(\mathrm{CE}_{\mathrm{aq}}\right)$ dos tratamentos e do $\mathrm{pH}$ foi de 1,85 e 11,60 dS m $\mathrm{dS}^{-1}$ e,33 e 6,40, respectivamente. Determinou-se a condutividade elétrica das soluções nutritivas utilizando-se o condutivímetro "Analyser - 600" enquanto o pH foi medido com o potenciômetro "Orion model 410A".

Após a aplicação dos tratamentos as plantas foram irrigadas por gotejamento em turno de rega diária, aplicando-se um volume de solução de aproximadamente $400 \mathrm{~mL}$, suficiente para substituir completamente o restante da irrigação anterior para 
o que se utilizou-se a mesma solução nutritiva, acrescida ou não de cloreto de sódio, conforme o tratamento.

O delineamento experimental utilizado foi inteiramente casualizado em um arranjo fatorial 10 x 2 (genótipos x níveis de salinidade), com três repetições por tratamento, totalizando 60 unidades experimentais, cada qual constituída de uma planta.

A produção relativa de biomassa seca da parte aérea das plantas (PR) foi determinada segundo metodologia proposta por Maas \& Hoffman (1977). O genótipo que apresentou produção relativa superior a $70 \%$ de sua testemunha no nível $100 \mathrm{~mol} \mathrm{~m}^{-3} \mathrm{de} \mathrm{NaCl}$ foi considerado tolerante dentro do grupo de genótipos estudados. Por outro lado, foram considerados sensíveis os genótipos que apresentaram produção relativa inferior a $50 \%$ de seus controles.

Para obtenção das variáveis de crescimento foram realizadas medições semanais do número de folhas (NF), mediante contagem; do diâmetro do pseudocaule (qPC) utilizando-se um paquímetro milimetrado (tendo como referência o local do pseudocaule identificado por uma fita colocada a $5 \mathrm{~cm}$ do nível do substrato); da altura da planta (ALT) com o auxílio de uma fita métrica (comprimento compreendido entre a fita referência e o ponto de inserção da $1^{\text {a }}$ folha totalmente expandida a partir da folha vela) e da área foliar (AF) multiplicando-se o produto da largura média do limbo foliar e o comprimento da folha pelo fator de correção 0,7 (Gomes et al., 2005b) expressa em cm².

Por ocasião da coleta, isto é, aos vinte e um dias de tratamento, foram coletados, separadamente, o limbo foliar, o pseudocaule e as raízes + rizoma e obtidas as biomassas frescas (limbo foliar - BFL, pseudocaule - BFPC e raízes + rizoma BFRR) utilizando-se uma balança digital com precisão de 0,01 g; em seguida, todo o material vegetal foi posto para secar em estufa de aeração forçada a $65{ }^{\circ} \mathrm{C}$ até peso constante, para posterior obtenção da biomassa seca (limbo foliar - BSL, pseudocaule - BSPC e raízes + rizoma - BSRR) utilizando-se a mesma balança digital.

Foram calculados, segundo Benincasa (2003):

$$
\begin{aligned}
& \text { Alocação de biomassa órgão }(\mathrm{AB})=\left(\mathrm{MS}_{\text {orgão }} / \mathrm{MS}_{\text {total }}\right) \times 100 ; \\
& \text { Suculência }_{\text {órão }}(\mathrm{SC})=\left(\mathrm{MF}_{\text {orgão }}-\mathrm{MS}_{\text {orgãoo }}\right) / \mathrm{MS}_{\text {orgão }} ; \\
& \text { Razão de área foliar }(\mathrm{RAF})=\mathrm{AF}_{\mathrm{f}} / \mathrm{MS}_{\mathrm{f}} ; \\
& \text { Taxa de crescimento absoluto }(\mathrm{TCA})=\left(\mathrm{ALT}_{\mathrm{f}}-\mathrm{ALT}_{\mathrm{i}}\right) / \mathrm{t} ; \\
& \text { Taxa de assimilação líquida } \\
& (\mathrm{TAL})=\left[\left(\ln _{\mathrm{AF}}-\ln \mathrm{AF}_{\mathrm{i}}\right) / \mathrm{t}\right] \times\left[\left(\mathrm{MS}_{\mathrm{f}}-\mathrm{MS}_{\mathrm{i}}\right) /\left(\mathrm{AF}_{\mathrm{f}}-\mathrm{AF}_{\mathrm{i}}\right)\right]
\end{aligned}
$$

\section{em que:}

$\mathrm{MS}_{\text {órgão }}$ - biomassa seca nos diferentes órgãos

$\mathrm{MS}_{\text {total }}$ - biomassa seca total

$\mathrm{MS}_{\text {folha }}$ - biomassa seca do limbo foliar

$\mathrm{MS}_{\mathrm{i}}$ - biomassa seca total inicial

$\mathrm{MS}_{\mathrm{f}}$ - biomassa seca total final

$\mathrm{MF}_{\text {órgão }}$ - biomassa fresca nos diferentes órgãos

$\mathrm{AF}_{\mathrm{i}}$ - área foliar inicial

$\mathrm{AF}_{\mathrm{f}}$ - área foliar final

$\mathrm{ALT}_{\mathrm{f}}$ - altura final da planta

$\mathrm{ALT}_{\mathrm{i}}$ - altura inicial da planta

BSPA - biomassa seca da parte aérea

BSRR - biomassa seca das raízes + rizoma e t é a duração dos tratamentos salinos, em dias
Os resultados das variáveis de crescimento obtidos foram analisados estatisticamente por meio do programa ASSISTAT (Silva \& Azevedo, 2002), análise de variância com teste F e aplicado o teste de Tukey a nível de $5 \%$ de probabilidade visando à comparação das médias. Para a variável alocação de biomassa nos diferentes órgãos, a análise de variância foi realizada utilizando-se a transformação arcosseno da raiz (X/ 100).

\section{RESULTADOS E DISCUSSÃO}

Os resultados referentes à produção relativa da biomassa seca da parte aérea (PR) dos 10 genótipos de bananeira diplóide, são apresentados na Tabela 2. Observa-se que a PR das plantas variou entre 41,01 e 74,22\% no nível $100 \mathrm{~mol} \mathrm{~m}^{-3} \mathrm{de} \mathrm{NaCl}$. Esta amplitude de variação indica que os genótipos testados possuem respostas diferenciadas ao estresse salino.

Tabela 2. Produção relativa da biomassa seca da parte aérea (PR) de 10 genótipos de bananeira diploide após 21 dias de estresse salino em condições de casa de vegetação

\begin{tabular}{lc}
\hline Genótipos & PR em \% (100 $\left.\mathbf{~ m o l ~}^{\mathbf{3}} \mathbf{~ d e ~} \mathbf{~ N a C l}\right)$ \\
Nyarmo Yik & 58,27 \\
T.D.Mak & 57,17 \\
Berlim & 57,00 \\
Pisang Ceylan & 58,46 \\
Tungia & 41,01 \\
Madu & 64,58 \\
Lidi & 74,22 \\
Ouro & 46,15 \\
Malbut & 61,05 \\
Calcuttá & 66,71 \\
\hline
\end{tabular}

Em $100 \mathrm{~mol} \mathrm{~m}^{-3}$ de $\mathrm{NaCl}$ a produção relativa da biomassa seca da parte aérea no genótipo Lidi, foi superior a $70 \%$, indicando maior aclimatação ao estresse. Por outro lado, nos genótipos Ouro e Tungia a produção relativa da biomassa seca foi inferior a $50 \%$, caracterizando sensibilidade, segundo classificação de Maas \& Hoffman (1977).

$\mathrm{A}$ adição de cloreto de sódio $(\mathrm{NaCl})$ à solução nutritiva provocou redução significativa no número de folhas mas apenas nos genótipos Thong Dok Mak, Berlim, Tungia e Madu na ordem de 12,87; 20,00; 17,27 e 14,25\%, respectivamente, em relação ao tratamento controle (Tabela 3 ). A redução do número de folhas em função do incremento de níveis de sal já foi descrita em outros genótipos de bananeira (Silva et al., 2009).

$\mathrm{O}$ diâmetro do pseudocaule (qPC) foi reduzido em quase todos os genótipos estudados merecendo destaque o genótipo Tungia, com redução da ordem de 26,36\% (Tabela 3). Com relação a qPC, os resultados obtidos neste trabalho corroboram com os de Neves et al. (2002) que mostraram reduções significativas no diâmetro do pseudocaule nas cultivares Nanicão e Prata quando submetidas a soluções salinas $(5,0$ a15,0 dS m ${ }^{-1}$.

A altura foi reduzida nas plantas submetidas ao estresse, parâmetro no qual merecem destaque os genótipos Ouro e Tungia, que apresentaram redução de altura da ordem de 38,74 
Tabela 3. Valores médios do número de folhas (NF), diâmetro do pseudocaule $(\theta P C)$, altura de plantas (ALT) e área foliar (AF) em genótipos diploides de bananeira submetidos ao estresse salino, no período de 21 dias

\begin{tabular}{|c|c|c|c|c|c|c|c|c|}
\hline \multirow{3}{*}{ Genótipos } & \multicolumn{8}{|c|}{ Variáveis biométricas/ Concentração de $\mathrm{NaCl}\left(\mathrm{mol} \mathrm{m}^{-3}\right)$} \\
\hline & \multicolumn{2}{|c|}{ NF } & \multicolumn{2}{|c|}{$\theta \mathrm{PC}(\mathrm{cm})$} & \multicolumn{2}{|c|}{ ALT $(\mathrm{cm})$} & \multicolumn{2}{|c|}{$A F\left(\mathrm{~cm}^{2}\right)$} \\
\hline & 0 & 100 & 0 & 100 & 0 & 100 & 0 & 100 \\
\hline Nyarmo Yik & 9,33 aABC & $8,67 \mathrm{aAB}$ & $2,04 \mathrm{aBC}$ & $1,73 \mathrm{bAB}$ & $26,00 \mathrm{aABC}$ & $18,83 \mathrm{bABC}$ & 2652,17 aABC & $1570,96 \mathrm{bAB}$ \\
\hline T.D.Mak & $10,33 \mathrm{aA}$ & 9,00 bA & $2,32 \mathrm{aAB}$ & $1,83 \mathrm{bAB}$ & $23,33 \mathrm{aABC}$ & $17,83 \mathrm{bABC}$ & 2363,17 aABCD & 1175,83 bB \\
\hline Berlim & $10,00 a A B$ & $8,00 \mathrm{bAB}$ & $2,12 \mathrm{aBC}$ & $1,73 \mathrm{bAB}$ & $18,83 \mathrm{aCD}$ & $14,50 \mathrm{aC}$ & 2126,15 aCD & 1121,11 bAB \\
\hline Tungia & 9,67 aAB & $8,00 \mathrm{bAB}$ & $2,20 a A B C$ & $1,62 \mathrm{bAB}$ & $28,00 a A B$ & $17,33 \mathrm{bABC}$ & 3060,35 aAB & $1878,61 \mathrm{bAB}$ \\
\hline Madu & $9,33 \mathrm{aABC}$ & $8,00 \mathrm{bAB}$ & $2,04 \mathrm{aBC}$ & $1,82 \mathrm{aAB}$ & 15,67 aD & $13,17 \mathrm{aC}$ & 2363,32 aABCD & $1706,71 \mathrm{bAB}$ \\
\hline P.Ceylan & $8,67 \mathrm{aABC}$ & $8,33 \mathrm{aAB}$ & $2,61 \mathrm{aA}$ & $2,08 \mathrm{bA}$ & $29,83 \mathrm{aA}$ & $24,67 \mathrm{bA}$ & 3109,83 aA & $2184,54 \mathrm{bA}$ \\
\hline Malbut & 8,33 aBC & 7,33 aAB & $1,82 \mathrm{aC}$ & $1,54 \mathrm{aB}$ & $21,83 \mathrm{aBCD}$ & $16,83 \mathrm{bBC}$ & $2192,01 \mathrm{aBCD}$ & $1396,58 \mathrm{bAB}$ \\
\hline Ouro & 8,67 aABC & 7,67 aAB & $1,91 \mathrm{aBC}$ & $1,55 \mathrm{bB}$ & $21,50 \mathrm{aBCD}$ & 13,17 bc & 2333,94 aABCD & 1124,79 bB \\
\hline Lidi & $9,00 \mathrm{aABC}$ & $8,67 \mathrm{aAB}$ & $1,90 \mathrm{aBC}$ & $1,71 \mathrm{aAB}$ & $23,50 \mathrm{aABC}$ & $22,33 a A B$ & $1500,12 \mathrm{aD}$ & 1140,31 bB \\
\hline Calcuttá & $7,67 \mathrm{aC}$ & $7,00 \mathrm{aB}$ & $1,96 \mathrm{aBC}$ & $1,67 \mathrm{bAB}$ & $20,33 \mathrm{aCD}$ & $15,73 \mathrm{bBC}$ & $2564,54 \mathrm{aABC}$ & $1918,12 \mathrm{bAB}$ \\
\hline Média & \multicolumn{2}{|c|}{8,580} & \multicolumn{2}{|c|}{1,910} & \multicolumn{2}{|c|}{20,160} & \multicolumn{2}{|c|}{1984,210} \\
\hline CV (\%) & \multicolumn{2}{|c|}{8,291} & \multicolumn{2}{|c|}{8,894} & \multicolumn{2}{|c|}{13,768} & \multicolumn{2}{|c|}{16,801} \\
\hline
\end{tabular}

probabilidade

e 38,11\%, respectivamente, enquanto o genótipo Lidi indicou a menor redução $(4,98 \%)$ (Tabela 3). A redução na altura de plantas de bananeira foi registrada previamente em genótipos triploides tendo a magnitude da variação oscilado entre 37,03 e 4,66\% (Gomes et al., 2005a). Segundo Benincasa (2003) em geral, a altura das plantas em geral é um dos parâmetros menos susceptíveis às variações ambientais. No caso do estresse salino isto parece ser bastante discutível considerando-se a redução da altura de sete genótipos neste trabalho, tal como no genótipo Prata, registrada por Neves et al. (2002).

Ocorreram reduções na área foliar em todos os genótipos estudados destacando-se os genótipos Thong Dok Mak e Ouro, com reduções acima de 50\%. Por outro lado foram verificadas, nos genótipos Lidi e Calcuttá, as menores reduções (23,99 e $25,21 \%$ respectivamente) em relação ao tratamento controle (Tabela 3). A redução da área foliar em função do incremento dos níveis de sal é uma resposta frequente em diversos genótipos de bananeira (Gomes et al., 2002, 2005b; Carmo et al., 2003; Silva et al., 2009; Willadino et al., 2011). A redução na taxa de assimilação líquida de $\mathrm{CO}_{2}$ provocada pelo fechamento dos estômatos em resposta ao baixo potencial da água do solo devido à elevada concentração salina (Gurgel et al., 2003) é também determinante na redução do crescimento foliar.

O genótipo Lidi foi o único sem redução significativa na biomassa fresca do limbo (BFL) do pseudocaule (BFPC) e total (BFT) enquanto nos genótipos Tungia e Ouro as reduções se situaram entre 54 e $62 \%$. O genótipo Lidi também não registrou redução na biomassa fresca da raiz (BFRR) da mesa forma que os genótipos Berlim e Madu. Mais uma vez o genótipo Tungia se destacou por apresentar maior redução nesta variável, da ordem de 52,81\% em relação ao tratamento controle (Tabela 4). Em geral, a diminuição da disponibilidade hídrica no solo ocasiona queda no potencial da água da folha levando à perda de turgescência e ao fechamento estomático, o que vai acarretar alterações na biomassa fresca do vegetal (Munns \& Tester, 2008).

A salinidade também provocou reduções significativas na biomassa seca, em todos os genótipos estudados nas variáveis: biomassa seca do limbo (BSL), pseudocaule (BSPC), raiz (BSRR) e total (BST) com exceção do genótipo Lidi. Os genótipos Tungia e Ouro apresentaram reduções superiores a $40 \%$. Na variável BSRR ocorreram reduções significativas nos

Tabela 4. Val ores médios da biomassa fresca (limbo foliar - BFL, pseudocaule - BFPC, raízes + rizoma - BFRR e total - BFT) em genótipos diploides de bananeira submetidos ao estresse salino pelo período de 21 dias

\begin{tabular}{|c|c|c|c|c|c|c|c|c|}
\hline \multirow{3}{*}{ Genótipos } & \multicolumn{8}{|c|}{ Variáveis biométricas/ Concentração de $\mathrm{NaCl}\left(\mathrm{mol} \mathrm{m}^{-3}\right)$} \\
\hline & \multicolumn{2}{|c|}{ BFL (g) } & \multicolumn{2}{|c|}{ BFPC (g) } & \multicolumn{2}{|c|}{ BFRR $(g)$} & \multicolumn{2}{|c|}{ BFT (g) } \\
\hline & 0 & 100 & 0 & 100 & 0 & 100 & 0 & 100 \\
\hline Nyarmo Yik & $79,31 \mathrm{aB}$ & $42,06 \mathrm{bAB}$ & $82,76 \mathrm{aC}$ & $40,10 \mathrm{bB}$ & $94,44 \mathrm{aA}$ & $64,60 \mathrm{bAB}$ & $256,65 \mathrm{aBC}$ & $146,75 \mathrm{bAB}$ \\
\hline T.D.Mak & $71,78 \mathrm{aBC}$ & 32,12 bB & $94,83 \mathrm{aBC}$ & $45,46 \mathrm{bB}$ & $91,09 \mathrm{aA}$ & $52,23 \mathrm{bABC}$ & $257,70 \mathrm{aBC}$ & $129,81 \mathrm{bAB}$ \\
\hline Berlim & $65,98 \mathrm{aBC}$ & $41,14 \mathrm{bAB}$ & $70,13 \mathrm{aC}$ & $40,77 \mathrm{bB}$ & $88,42 \mathrm{aAB}$ & 74,13 aA & $224,53 \mathrm{aBC}$ & $156,03 \mathrm{bAB}$ \\
\hline Tungia & $85,12 \mathrm{aB}$ & 36,84 bB & $123,67 \mathrm{aAB}$ & $47,00 \mathrm{bB}$ & 87,80 aAB & $41,43 \mathrm{bBC}$ & $296,60 \mathrm{aAB}$ & $125,27 \mathrm{bAB}$ \\
\hline Madu & $86,59 \mathrm{aB}$ & $51,10 \mathrm{bAB}$ & $90,78 \mathrm{aBC}$ & $52,11 \mathrm{AB}$ & $51,64 \mathrm{aC}$ & $38,76 \mathrm{aBC}$ & $229,01 \mathrm{aBC}$ & $141,97 \mathrm{bAB}$ \\
\hline P.Ceylan & 120,87 aA & 67,28 bA & 152,83 aA & 85,77 bA & 82,39 aAB & $50,49 \mathrm{bABC}$ & 356,10 aA & 203,53 bA \\
\hline Malbut & $66,18 \mathrm{aBC}$ & 37,26 bB & $63,18 \mathrm{aC}$ & 36,75 bB & $61,43 \mathrm{aBC}$ & 39,56 bBC & $190,79 \mathrm{aC}$ & $113,57 \mathrm{bB}$ \\
\hline Ouro & 76,19 aB & $32,00 \mathrm{bB}$ & $84,20 \mathrm{aC}$ & 36,35 bB & $59,08 \mathrm{aBC}$ & 33,19 bC & $219,47 \mathrm{aBC}$ & 101,54 bB \\
\hline Lidi & $44,58 \mathrm{aC}$ & $29,80 \mathrm{aB}$ & $75,72 \mathrm{aC}$ & $53,94 \mathrm{aAB}$ & $48,58 \mathrm{aC}$ & $40,16 \mathrm{aBC}$ & $168,89 \mathrm{aC}$ & $123,90 \mathrm{aAB}$ \\
\hline Calcuttá & $71,80 \mathrm{aBC}$ & $52,62 \mathrm{bAB}$ & $78,19 \mathrm{aC}$ & 47,54 bB & $72,94 \mathrm{aABC}$ & $46,53 \mathrm{bABC}$ & $222,93 \mathrm{aBC}$ & $146,70 \mathrm{bAB}$ \\
\hline Média & \multicolumn{2}{|c|}{59,530} & \multicolumn{2}{|c|}{70,100} & \multicolumn{2}{|c|}{60,940} & \multicolumn{2}{|c|}{190,590} \\
\hline CV (\%) & \multicolumn{2}{|c|}{18,237} & \multicolumn{2}{|c|}{19,767} & \multicolumn{2}{|c|}{17,834} & \multicolumn{2}{|c|}{17,202} \\
\hline
\end{tabular}

Letras minúsculas iguais entre os níveis de salinidade para o mesmo genótipo e maiúsculas entre os genótipos no mes mo nível de salinidade não diferem entre si pelo teste de Tukeya nível de 0,05 de probabilidade 
genótipos Thong Dok Mak, Tungia, Pisang Ceylan, Ouro e Calcuttá superiores a 36\%. Os demais genótipos não sofreram alterações para esta variável (Tabela 5). Com base em trabalhos com bananeira, a biomassa seca se tem destacado como uma das variáveis mais importantes. Nesses trabalhos foram observadas reduções na produção de biomassa seca em diversas cultivares estudadas quando submetidas a $100 \mathrm{~mol} \mathrm{~m}^{-3}$ de $\mathrm{NaCl}$ (Gomes et al., 2005b; Silva et al., 2009; Willadino et al., 2011). A redução da biomassa é consequência da redução da taxa fotossintética e do desvio de energia destinada ao crescimento para a ativação e manutenção de atividade metabólica associada à adaptação à salinidade como a manutenção da integridade das membranas, síntese de solutos orgânicos para a osmorregulação e/ou proteção de macromoléculas e a regulação do transporte e distribuição iônica em vários órgãos e dentro das células (Munns et al., 2002).

A salinidade provocou redução significativa na alocação da biomassa do limbo (ABL) nos genótipos Nyarmo Yik, Berlim, Ouro e Lidi, da ordem de 10,79, 13,62, 11,85 e 8,76\%, respectivamente, em relação ao tratamento controle. No que se refere à alocação da biomassa das raízes + rizoma (ABRR) ocorreu um incremento da ordem de 29,61 e 22,96\% nos genótipos Nyarmo Yik e Berlim, respectivamente (Tabela 6) favorecendo a absorção de água e nutrientes minerais. De maneira geral, os genótipos de bananeira crescidos em condições de estresse salino aumentaram a alocação de biomassa nas raízes com consequente redução nas folhas (Tabela 6). Entre os órgãos, entretanto, as folhas tiveram a maior participação percentual com relação à biomassa seca total da planta. A literatura referente à alocação de biomassa em plantas cultivadas, principalmente em bananeira sob estresse salino, é bastante escassa, não obstante sua importância para o estudo da translocação dos fotoassimilados entre os diversos órgãos da planta.

A salinidade também provocou redução significativa na suculência do limbo (SCL) no genótipo Thong Dok Mak, da ordem de 24,57\%; apesar disto, não se observou alteração significativa com relação à SCRR em nenhum genótipo estudado (Tabela 6). Observou-se, na maioria dos genótipos, maior suculência nas raízes + rizoma do que no limbo foliar.

Tabela 5. Valores médios da biomassa seca (limbo foliar - BSL, pseudocaule - BSPC, raízes + rizoma - BSRR e total BST) em genótipos diploides de bananeira submetidos ao estresse salino pelo tempo de 21 dias

\begin{tabular}{|c|c|c|c|c|c|c|c|c|}
\hline \multirow{3}{*}{ Genótipos } & \multicolumn{8}{|c|}{ Variáveis biométricas/ Concentração de $\mathrm{NaCl}\left(\mathrm{mol} \mathrm{m}^{-3}\right)$} \\
\hline & \multicolumn{2}{|c|}{ BSL (g) } & \multicolumn{2}{|c|}{ BSPC $(g)$} & \multicolumn{2}{|c|}{ BSRR $(g)$} & \multicolumn{2}{|c|}{ BST (g) } \\
\hline & 0 & 100 & 0 & 100 & 0 & 100 & 0 & 100 \\
\hline Nyarmo Yik & $10,16 \mathrm{aB}$ & $5,88 \mathrm{bAB}$ & $4,11 \mathrm{aC}$ & $2,43 \mathrm{bB}$ & $4,65 \mathrm{aAB}$ & $4,05 \mathrm{aAB}$ & 18,91 aABC & $12,36 \mathrm{bA}$ \\
\hline T.D.Mak & $9,00 \mathrm{aBC}$ & $5,14 \mathrm{bAB}$ & $4,65 \mathrm{aBC}$ & $2,67 \mathrm{bB}$ & $4,64 \mathrm{aAB}$ & $2,93 \mathrm{bBC}$ & $18,30 \mathrm{aABC}$ & $10,74 \mathrm{bA}$ \\
\hline Berlim & $8,18 \mathrm{aBC}$ & $5,30 \mathrm{bAB}$ & $3,67 \mathrm{aC}$ & $2,64 \mathrm{aB}$ & $5,39 a A$ & $5,11 \mathrm{aA}$ & $17,24 \mathrm{aBCD}$ & $13,05 \mathrm{bA}$ \\
\hline Tungia & $10,68 \mathrm{aAB}$ & $4,34 \mathrm{bAB}$ & $6,13 \mathrm{aAB}$ & $2,54 \mathrm{bB}$ & 3,49 aABC & $1,61 \mathrm{bC}$ & $20,30 \mathrm{aAB}$ & $8,49 \mathrm{bA}$ \\
\hline Madu & $9,96 \mathrm{aB}$ & $6,37 \mathrm{bAB}$ & $4,75 \mathrm{aBC}$ & $3,13 \mathrm{bAB}$ & $2,37 \mathrm{aC}$ & $1,69 \mathrm{aC}$ & $17,04 \mathrm{aBCD}$ & $11,19 \mathrm{bA}$ \\
\hline P.Ceylan & $13,57 \mathrm{aA}$ & $7,47 \mathrm{bA}$ & $6,77 \mathrm{aA}$ & 4,76 bA & $3,31 \mathrm{aBC}$ & $1,93 \mathrm{bC}$ & $23,65 \mathrm{aA}$ & $13,82 \mathrm{bA}$ \\
\hline Malbut & $7,96 \mathrm{aBC}$ & $4,72 \mathrm{bAB}$ & $3,31 \mathrm{aC}$ & $2,15 \mathrm{bB}$ & $2,63 \mathrm{aBC}$ & $1,91 \mathrm{aC}$ & $13,90 \mathrm{aCD}$ & $8,79 \mathrm{bA}$ \\
\hline Ouro & $8,89 \mathrm{aBC}$ & 3,84 bB & $4,22 \mathrm{aC}$ & $2,21 \mathrm{bB}$ & $3,16 \mathrm{aBC}$ & $1,65 \mathrm{bC}$ & $16,27 \mathrm{aBCD}$ & $7,70 \mathrm{bA}$ \\
\hline Lidi & $5,83 \mathrm{aC}$ & $4,11 \mathrm{aB}$ & $3,52 \mathrm{aC}$ & $2,83 \mathrm{aB}$ & $2,40 \mathrm{aC}$ & $2,13 \mathrm{aBC}$ & $11,75 \mathrm{aD}$ & $9,07 \mathrm{aA}$ \\
\hline Calcuttá & $9,70 \mathrm{aB}$ & $6,39 \mathrm{bAB}$ & $4,81 \mathrm{aBC}$ & $3,29 \mathrm{bAB}$ & $3,55 a A B C$ & $2,28 \mathrm{bBC}$ & 18,06 aABC & $11,96 \mathrm{bA}$ \\
\hline Média & \multicolumn{2}{|c|}{7,370} & \multicolumn{2}{|c|}{3,730} & \multicolumn{2}{|c|}{3,040} & \multicolumn{2}{|c|}{14,130} \\
\hline CV $(\%)$ & \multicolumn{2}{|c|}{16,199} & \multicolumn{2}{|c|}{17,234} & \multicolumn{2}{|c|}{24,515} & \multicolumn{2}{|c|}{16,269} \\
\hline
\end{tabular}

Letras minúsculas iguais entre os níveis de salinidade para o mesmo genótipo e maiúsculas entre os genótipos no mesmo nível de salinidade não diferem entre si pelo teste de Tukeya nível de 0,05 de probabilidade

Tabela 6. Valores médios da alocação de biomassa (limbo foliar - ABL e raízes + rizoma - ABRR) e suculência (limbo foliar - SCL e raízes + rizoma - SCRR) em genótipos diploides de bananeira submetidos ao estresse salino pelo período de 21 dias

\begin{tabular}{|c|c|c|c|c|c|c|c|c|}
\hline \multirow{3}{*}{ Genótipos } & \multicolumn{8}{|c|}{ Variáveis biométricas/Concentração de $\mathrm{NaCl}\left(\mathrm{mol} \mathrm{m}^{-3}\right)$} \\
\hline & \multicolumn{2}{|c|}{$A B L(\%)$} & \multicolumn{2}{|c|}{ ABRR (\%) } & \multicolumn{2}{|c|}{$\mathrm{SCL}\left(\mathrm{g} \mathrm{H}_{2} \mathrm{Og}^{-1} \mathrm{MS}\right)$} & \multicolumn{2}{|c|}{ SCRR ( $\left.\mathrm{g} \mathrm{H}_{2} \mathrm{Og}^{-1} \mathrm{MS}\right)$} \\
\hline & 0 & 100 & 0 & 100 & 0 & 100 & 0 & 100 \\
\hline Nyarmo Yik & 53,86 aABC & $48,05 \mathrm{bBC}$ & $24,35 \mathrm{bABC}$ & $31,56 a A B$ & $6,82 \mathrm{aA}$ & $6,20 \mathrm{aBC}$ & 19,44 aABC & $16,08 \mathrm{aCD}$ \\
\hline T.D.Mak & $49,31 \mathrm{aBC}$ & $47,94 \mathrm{aBC}$ & 25,11 aAB & $27,15 \mathrm{aBC}$ & 6,96 aA & $5,25 \mathrm{bC}$ & $18,97 \mathrm{aABC}$ & $16,91 \mathrm{aBCD}$ \\
\hline Berlim & $47,37 \mathrm{aC}$ & 40,92 bD & 31,40 bA & 38,61 aA & $7,07 \mathrm{aA}$ & 6,74 aABC & $15,47 \mathrm{aC}$ & 13,88 aD \\
\hline Tungia & 52,59 aABC & 51,30 aABC & 17,19 aCD & $18,54 \mathrm{aDEF}$ & $6,92 \mathrm{aA}$ & 7,47 abA & $24,00 \mathrm{aA}$ & $25,22 \mathrm{aA}$ \\
\hline Madu & 58,33 aA & 56,99 aA & 13,73 aD & $14,94 \mathrm{aEF}$ & $7,69 \mathrm{aA}$ & $7,02 \mathrm{aAB}$ & 21,10 aABC & $22,33 \mathrm{aAB}$ \\
\hline P.Ceylan & $57,39 \mathrm{aA}$ & 53,96 aAB & $14,01 \mathrm{aD}$ & $14,07 \mathrm{aF}$ & 7,91 aA & $8,02 \mathrm{aA}$ & 23,88 aAB & 25,17 aA \\
\hline Malbut & 57,27 aA & $53,87 \mathrm{aAB}$ & $18,93 \mathrm{aBCD}$ & $21,81 \mathrm{aCDE}$ & $7,33 \mathrm{aA}$ & $6,85 \mathrm{aAB}$ & $22,36 \mathrm{aAB}$ & $19,67 \mathrm{ABCD}$ \\
\hline Ouro & $54,60 a A B$ & $48,13 \mathrm{bBC}$ & $19,35 \mathrm{aBCD}$ & $21,91 \mathrm{aCD}$ & 7,59 aA & 7,39 aAB & $18,05 \mathrm{aBC}$ & 20,47 aABC \\
\hline Lidi & $49,56 \mathrm{aBC}$ & $45,22 b C D$ & $20,26 \mathrm{aBCD}$ & 23,55 aCD & 6,58 aA & $6,24 \mathrm{aBC}$ & 19,18 aABC & 17,83 aBCD \\
\hline Calcuttá & 53,68 aABC & $53,44 \mathrm{aAB}$ & $19,64 \mathrm{aBCD}$ & 19,08 aDEF & $6,38 \mathrm{aA}$ & $7,23 \mathrm{aAB}$ & $19,65 \mathrm{aABC}$ & $19,47 \mathrm{aABCD}$ \\
\hline Média & \multicolumn{2}{|c|}{45,970} & \multicolumn{2}{|c|}{27,590} & \multicolumn{2}{|c|}{6,980} & \multicolumn{2}{|c|}{19,960} \\
\hline CV (\%) & \multicolumn{2}{|c|}{3,220} & \multicolumn{2}{|c|}{6,844} & \multicolumn{2}{|c|}{8,198} & \multicolumn{2}{|c|}{10,902} \\
\hline
\end{tabular}

Letras minúsculas iguais entre os níveis de salinidade para o mesmo genótipo e maiúsculas entre os genótipos no mesmo nível de salinidade, não diferem entre si pelo teste de Tukeya nível de 0,05 de probabilidade 
Tabela 7. Valores médios da razão de área foliar (RAF), taxa de assimilação líquida (TAL) e taxas de crescimento absoluto (TCA) em genótipos diploides de bananeira submetidos ao estresse salino durante 21 dias

\begin{tabular}{|c|c|c|c|c|c|c|}
\hline \multirow{3}{*}{ Genótipos } & \multicolumn{6}{|c|}{ Variáveis biométricas/Concentração de $\mathrm{NaCl}\left(\mathrm{mol} \mathrm{m}^{-3}\right)$} \\
\hline & \multicolumn{2}{|c|}{$\operatorname{RAF}\left(\mathrm{cm}^{2} \mathrm{~g}^{-1} \mathrm{MS}\right)$} & \multicolumn{2}{|c|}{ TAL $\left(m g M S \mathrm{~cm}^{-2} \mathrm{~d}^{-1}\right)$} & \multicolumn{2}{|c|}{$\mathrm{TCA}\left(\mathrm{mm} \mathrm{d}^{-1}\right)$} \\
\hline & 0 & 100 & 0 & 100 & 0 & 100 \\
\hline Nyarmo Yik & $262,07 \mathrm{aA}$ & $275,27 \mathrm{aB}$ & $0,21 \mathrm{aA}$ & $0,12 \mathrm{bA}$ & 5,77 aABC & $2,17 \mathrm{bAB}$ \\
\hline T.D. MAK & $263,78 \mathrm{aA}$ & $229,33 \mathrm{aB}$ & $0,23 \mathrm{aA}$ & $0,12 \mathrm{bA}$ & $5,43 \mathrm{aABCD}$ & $2,73 \mathrm{bAB}$ \\
\hline Berlim & $263,24 \mathrm{aA}$ & $250,03 \mathrm{aB}$ & $0,19 \mathrm{aA}$ & $0,08 \mathrm{bA}$ & 4,30 aABCD & $1,63 \mathrm{bAB}$ \\
\hline Tungia & $290,68 \mathrm{bA}$ & $423,04 \mathrm{aA}$ & $0,19 \mathrm{aA}$ & $0,09 \mathrm{bA}$ & $6,57 \mathrm{aA}$ & $1,83 \mathrm{bAB}$ \\
\hline Madu & 238,33 aA & $269,97 \mathrm{aB}$ & $0,18 \mathrm{aA}$ & $0,13 \mathrm{bA}$ & $3,00 \mathrm{aD}$ & $1,67 \mathrm{aAB}$ \\
\hline P.Ceylan & $230,02 \mathrm{aA}$ & $295,22 \mathrm{aB}$ & $0,23 \mathrm{aA}$ & $0,12 \mathrm{bA}$ & $6,43 \mathrm{aAB}$ & $3,90 \mathrm{bA}$ \\
\hline Malbut & $276,53 \mathrm{aA}$ & $294,87 \mathrm{aB}$ & $0,17 \mathrm{aA}$ & $0,09 \mathrm{bA}$ & $3,73 \mathrm{aCD}$ & $0,93 \mathrm{bB}$ \\
\hline Ouro & $263,09 \mathrm{aA}$ & $302,43 \mathrm{aB}$ & $0,18 \mathrm{aA}$ & $0,07 \mathrm{bA}$ & $3,97 \mathrm{aBCD}$ & $1,17 \mathrm{bB}$ \\
\hline Lidi & $258,67 \mathrm{aA}$ & $279,40 \mathrm{aB}$ & $0,22 \mathrm{aA}$ & $0,10 \mathrm{bA}$ & 4,10 aABCD & $2,60 \mathrm{aAB}$ \\
\hline Calcuttá & $261,53 \mathrm{aA}$ & $300,79 \mathrm{aB}$ & $0,18 \mathrm{aA}$ & $0,10 \mathrm{bA}$ & 4,37 aABCD & $2,07 \mathrm{bAB}$ \\
\hline Média & \multicolumn{2}{|c|}{276,410} & \multicolumn{2}{|c|}{0,150} & \multicolumn{2}{|c|}{3,420} \\
\hline CV $(\%)$ & \multicolumn{2}{|c|}{15,675} & \multicolumn{2}{|c|}{16,858} & \multicolumn{2}{|c|}{27,068} \\
\hline
\end{tabular}

Letras minúsculas iguais entre os níveis de salinidade para o mesmo genótipo e maiúsculas entre os genótipos no mesmo nível de salinidade, não diferem entre si pelo teste de Tukeya nível de 0,05 de probabilidade

Constatou-se uma redução na taxa de assimilação líquida (TAL) em que todos os genótipos estudados destacando-se o genótipo Ouro, com redução da ordem de 61,11\% (Tabela 7).

Quanto à RAF, a salinidade provocou um incremento significativo apenas no genótipo Tungia, da ordem de $45,53 \%$, em função da grande redução na produção de biomassa seca; nos demais genótipos o incremento da salinidade na solução nutritiva não produziu efeito significativo na razão de área foliar, sinalizando que o efeito do estresse salino na área foliar foi da mesma magnitude que na produção de biomassa seca (Tabela 7).

Na taxa de crescimento absoluto (TCA) a salinidade provocou reduções significativas na maioria dos genótipos estudados, com exceção dos genótipos Madu e Lidi. Este último genótipo apresentou uma redução de apenas 36,6\% refletindo sua capacidade de adaptação ao estresse salino, como constatado em diversas variáveis de crescimento já discutidas. Em contraste, os genótipos Tungia e Ouro apresentaram reduções superiores a 70\% caracterizando, mais uma vez, sensibilidade ao estresse salino.

\section{CONCLUSÕES}

1. O genótipo Lidi possui maior tolerância à salinidade e, portanto, genes de interesse para o melhoramento genético

2. Os genótipos Ouro e Tungia são os mais sensíveis ao estresse salino.

\section{LITERATURA CITADA}

Aslam, M.; Qureshi, R. H.; Ahmad, N. A rapid screening technique for sal tolerance in rice (Oryza sativa $\mathrm{L}$.). Plant and Soil, v.150, p-99-107, 1993.

Benincasa, M. M. P. Análise de crescimento de plantas (noções básicas). Jaboticabal: FUNEP, 2003.41p.

Carmo, G. A.; Medeiros, J. F. de; Tavares, J. C. Crescimento de bananeiras sob diferentes níveis de salinidade da água de irrigação. Revista Brasileira deFruticultura, v.25, p.513-518, 2003.
Dasgan, H. Y.; Aktas, H.; Abak, K.; Cakmak, I.Determination of screening techniques to salinity tolerance in tomatoes and investigation of genotype responses. Plant Science, v.163, p.695-703, 2002.

Fageria, N. K.; Soares Filho, W. S.; Gheyi, H. R. In: Gheyi, H. R.; Dias, N. S.; Lacerda, C. F. (ed.). Manejo da salinidade na agricultura. Fortaleza: INCT Sal, 2010.p.206-217.

FAO/STAT. Produção Mundial de Banana. http://www.fao.org. 28 Out. 2011.

Gomes, E. W. F. Willadino, L.; Martins, L. S. S.; Câmara, T. R. Variedades de bananeira tratadas com água salinizada em fase inicial de crescimento. Revista Brasileira de Engenharia Agrícola e Ambiental, v.9, p.31-36, 2005a.

Gomes, E. W. F.; Willadino, L.; Martins, L. S. S.; Camara, T. R.; Silva, S. O. Banana genotypes under salt stress: Tolerance and sensitivity. Infomusa, v.11, p.13-18, 2002.

Gomes, E. W. F. Willadino, L; Martins, L. S. S.; Silva, S. O.; Câmara, T. R. Variabilidade genética de genótipos de bananeira (Musa spp.) submetidos ao estresse salino. Revista Brasileira de Engenharia Agrícola e Ambiental, v.9, p.171-177, 2005b.

Gomes, E. W. F; Willadino, L.; Martins, L. S. S.; Silva, S. O.; Camara, T. R.; Meunier, I. M. J. Diplóides (AA) de bananeira submetidos ao estresse salino. Pesquisa Agropecuária Brasileira, v.39, p.525-531, 2004.

Gurgel, M. T.; Fernandes, P. D.; Gheyi, H. R.; Santos, F. J. S.; Bezerra, I. L.; Nobre, R.G. Estresse salino na germinação e formação de porta-enxerto de aceroleira. Revista Brasileira de Engenharia Agrícola e Ambiental, v.7, p.31-36, 2003.

Maas, E. V.; Hoffman, G. J. Crop salt tolerance: current assessment. Journal of Irrigation and Drainage, v.103, p.115134, 1977.

Munns, R.; Husain, S.; Rivelli, A. R.; Richard, A. J.; Condon, A. G.; Megan, P. L.; Evans, S. L.; Schachtman, D. P.; Hare, R. A.Avenues for increasing salt tolerance of crops, and the roleof physiologically based selection traits. Plant and Soil, v.247,p.93-105, 2002.

Munns, R.; Tester, M. Mechanisms of salinity tolerance. The Annual Review of Plant Biology, v.59, p.651-81, 2008. 
Neves, L. L. de M.; Siqueira, D. L. de; Cecon, P. R.; Martinez, C. A.; Salomão, L. C. C. Crescimento, trocas gasosas e potencial osmótico da bananeira-'prata', submetida a diferentes doses de sódio e cálcio em solução nutritiva. Revista Brasileira de Fruticultura, v.24, p.524-529, 2002.

Silva, F. A. S. e; Azevedo, C. A. V. de. Versão do programa computacional Assistat para osistema operacional Windows. Revista Brasileira de Produtos Agroindustriais, v.4, p.71-78, 2002.

Silva Neto, S. P. da; Guimarães, T. G. Evolução da cultura da banana no Brasil e no mundo. http://www.cpac.embrapa.br/ noticias/artigosmidia/publicados/287. 28 Out. 2011.
Silva, R. L. O.; Martins, L. S. S.; Gomes, E. W. F.; Ferraz, G. M. G.; Silva, S. O.; Willadino, L. Avaliação de diplóides de bananeira (Musa spp.) quanto à tolerância a salinidade. Revista Brasileira de Fruticultura, v.31, p.1084-1091, 2009.

Silva, S. O.; Carvalho, P. C. L.; Shepherd, K; Alves, E. J.; Oliveira, C. A. P.; Carvalho, J. A. B. S. Catálogo de germoplasma de bananeira (Musa spp). Cruz das Almas: Embrapa Mandioca e Fruticultura, 1999. 152p.

Willadino, L.;.Gomes, E. W. F.; Silva, E. F. F.; Martins, L. S. S.; Camara, T. R. Efeito do estresse salino em genótipos tetraplóides de bananeira. Revista Brasileira de Engenharia Agrícola e Ambiental, v.15, p.53-59, 2011. 